\title{
Gauge Invariance and the Generalized Bondi-Metzner Algebra
}

\author{
MAYER Humi \\ Department of Mathematics, University of Toronto, Toronto, Canada
}

Received January 20, 1971

Abstract. We examine the algebraic meaning of the Electromagnetic gauge invariance and show that it leads to the new concepts of gauged operators, gauged representations and hence to infinite dimensional extensions of Lie algebras. In particular we prove that the generalized Bondi-Metzner algebra can be interpreted as a gauged Lorentz algebra related to the Electromagnetic gauge.

\section{Introduction}

It is well known that Quantum Mechanical states are phase invariant. The reason for this fact is that in Quantum Mechanics the state functions are not directly measurable quantities but only the absolute value of the scalar product, $|(f, g)|$ which is invariant under the replacements

$$
f_{1}=e^{i \theta} f, g_{1}=e^{i \psi} g \quad(\theta, \psi \text { reals }) .
$$

As a result of this invariance we have to consider in Quantum Mechanics rays of functions and therefore also operator rays and ray representations of Lie algebras $[1,2]$.

However in physics we deal with another important case in which we use quantities which are not directly measurable. This case is the vector potential formalism of the Electromagnetic field. As in the quantum case this formalism is not determined uniquely by the measurable quantities and this enables us to gauge the vector potentials by quantities of the form

$$
\left(\operatorname{grad} f, \frac{\partial f}{\partial t}\right)
$$

( $f$ well behaved function).

In this paper we show that the analogy between the phase invariance of the quantum wave functions and the gauge invariance of the Electromagnetic potentials can be pushed further and lead us to consider naturally, the new concepts of gauged operators, gauged representations and hence infinite dimensional extensions of Lie algebras. 
The plan of the paper is as follows:

In Section 2 we introduce the new mathematical concepts of the theory. In Section 3 we specialize to the Electromagnetic case and prove that one of the possible one-gauged Lorentz algebra is isomorphic to the generalized Bondi-Metzner algebra [3, 4].

We remark here that the generalized Bondi-Metzner algebra was first found as a formal asymptotic symmetry group of the gravitational field equations and many attempts were made to understand this result or to impose further conditions which will reduce it to the Poincare algebra $[4,5]$. Thus, besides the intrinsic interest of this work, it provides a new interpretation for the Bondi-Metzner algebra and implies a possible new connection between the Gravitational and Electromagnetic fields.

\section{Gauged Representations}

To start with we restate the meaning of the Electromagnetic gauge invariance in the language of differential forms.

To this end we remark that Maxwell's equations in free space can be written in the form ${ }^{1}$

where $\alpha$ is the two-form

$$
d \alpha=0, \quad d * \alpha=0
$$

$\alpha=\left(E_{1} d x^{1}+E_{2} d x^{2}+E_{3} d x^{3}\right) d t+\left(H_{1} d x^{2} d x^{3}+H_{2} d x^{3} d x^{1}+H_{3} d x^{1} d x^{2}\right)$

and the Electromagnetic potential $(\underline{A}, \phi)$ can be written as a one-form

$$
\lambda=\underline{A} \cdot d \underline{x}+\phi d t
$$

where the relation between $\alpha$ and $\lambda$ is

$$
d \lambda=\alpha .
$$

From this last relation it is evident that although it is more convenient to work with the potential $\lambda$ a physical (measurable) meaning is associated to the exterior derivative $d \lambda$ only.

To deal with the general case let $X$ be a Riemanian manifold and $C$ the space of differential forms on $X$

$$
C=\Sigma \oplus C^{p}
$$

where $C^{p}$ is the space of $p$-forms on $X$. To define the analog of a ray of functions we consider the direct sum $C^{p} \oplus R$ (i.e. the pairs $(\lambda, c)$ where $\left.\lambda \in C^{p}, c \in R\right)$ and define;

\footnotetext{
${ }^{1}$ We employ the usual notations of differential forms theory see [6].
} 
Definition 1. Let $\lambda \in C^{p}$, the gauged ray of $\lambda$ in $C^{p} \oplus R$ is

$$
\underline{\lambda}=\left\{(\lambda+d f, c) ; f \in C^{p-1}, c \in R\right\} .
$$

Thus the gauged rays in $C^{p} \oplus R$ are the cosets of the vector space

$$
M^{p}=C^{p} \oplus R / d C^{p-1} \oplus R
$$

$\left(C^{p} \oplus R\right.$ and $d C^{p-1} \oplus R$ are considered in (2.7) as real vector spaces). Similarly the gauged rays of $C \oplus R=\Sigma \oplus\left(C^{p} \oplus R\right)$ are the cosets of $C \oplus R / d C \oplus R=\Sigma \oplus M^{p}$.

To keep the physical background we remark that the consideration of the gauged rays in $C^{p} \oplus R$ rather than $C^{p}$ only has no influence on our motivation. In fact the natural way to define an exterior derivative $\bar{d}$ on $C \oplus R$ is as follows

$$
\bar{d}(\lambda, c)=(d \lambda, 0)
$$

and hence the physical content of the theory (which is related to $d \lambda$ only) is preserved.

Let $P(X)$ be the following set of operators in $\operatorname{Hom}_{R}(C)$

$$
P(X)=\left\{L \in \operatorname{Hom}_{R}(C) \mid \begin{array}{ll}
1 . & L \text { linear graded of degree zero } \\
\text { 2. } & d L=L d
\end{array}\right\} .
$$

From now on we consider only such operators in $\operatorname{Hom}_{R}(C)$. It is easy to verify the following

Lemma 1. a) $P(X)$ is a subalgebra in $\operatorname{Hom}_{R}(C)$.

b) $P(X)$ is a Lie subalgebra in the Lie algebra of $\operatorname{Hom}_{R}(C)$.

We remark that if $\phi$ is a smooth map $\phi: X \rightarrow X$ then $\phi^{*} \in P(X)$ (where $\phi^{*}$ is the induced map on $C$ ).

We define now the $p$-gauged operator $(L, d f), L \in P(X) f \in C^{p-1}$

as

$$
(L, d f): C^{p} \oplus R \rightarrow C^{p} \oplus R
$$

$$
(L, d f)(\lambda, c)=(L \lambda+c d f, 0) .
$$

Lemma 2. $(L, d f) \in \operatorname{Hom}_{R}\left(C^{p} \oplus R\right)$. The set of p-gauged operators in $\operatorname{Hom}_{R}\left(C^{p} \oplus R\right)$ (designated by $O\left(C^{p} \oplus R\right)$ ) with the operations

$$
\begin{aligned}
\left(L_{1}, d f_{1}\right)+\left(L_{2}, d f_{2}\right) & =\left(L_{1}+L_{2}, d\left(f_{1}+f_{2}\right)\right) \\
\left(L_{1}, d f_{1}\right)\left(L_{2}, d f_{2}\right) & =\left(L_{1} L_{2}, d L_{1} f_{2}\right)
\end{aligned}
$$

form a closed subalgebra of $\operatorname{Hom}_{R}\left(C^{p} \oplus R\right)$ and a sub-Lie algebra of the Lie algebra of $\mathrm{Hom}_{R}\left(C^{p} \oplus R\right)$.

Proof. By direct verification. 
We are now ready to define the concept of a gauged ray of operators.

Definition 2. Let $L \in P(X)$, the $p$-gauged ray of $L$ in $\operatorname{Hom}_{R}\left(C^{p} \oplus R\right)$ is

$$
\underline{L}=\left\{(L, d f) ; f \in C^{p-1}\right\}
$$

As can be easily verified the action of $(L, d f)$ on $C^{p} \oplus R$ induce a well defined action on $M^{p}$ i.e. $(L, d f) \underline{\lambda} \in \underline{L \lambda}$. Moreover if $(L, d f)$ is any representative of $\underline{L}$ and $\lambda$ any representative of $\underline{\lambda}$ then the image $(L, d f) \lambda$ in $M^{p}$ is independent of the choice of the representatives and is always in $\underline{L \lambda}$ thus we may write

$$
\underline{L} \cdot \underline{\lambda}=\underline{L \lambda}
$$

(and this action is linear in $M^{p}$ ). It is also easy to check that

$$
\underline{L}_{1}+\underline{L}_{2}=\underline{L_{1}+L_{2}}, \underline{L}_{1} \cdot \underline{L}_{2}=\underline{L_{1} L_{2}}
$$

and so we have proved the following.

Lemma 3. For any $L \in P(X), \underline{L} \in \operatorname{Hom}_{R}\left(M^{p}\right)$. Moreover the set of p-gauged ray operators (designated by $O\left(M^{p}\right)$ ) forms a closed subalgebra in $\operatorname{Hom}_{R}\left(M^{p}\right)$ and a subLie algebra of the Lie algebra of $\operatorname{Hom}_{R}\left(M^{p}\right)$.

We may therefore make the following definition:

Definition 3. A p-gauged representation of a Lie algebra $G$ is a homomorphism of $G$ into the Lie algebra of $O\left(M^{p}\right)$ i.e. a map $A \rightarrow \underline{L}(A), A \in G$, such that

$$
[\underline{L}(A), \underline{L}(B)]=\underline{L}([A B]) .
$$

We now prove the following.

Theorem 1. Let $\underline{L}^{R}(G)$ be a p-gauged representation of $G$ in $O\left(M^{p}\right)$ then there exist an (infinite dimensional) extension $\underline{G}$ of $G$ and a representation $L^{R}(\underline{G})$ in $O\left(C^{p} \oplus R\right)$ such that the following diagram is commutative

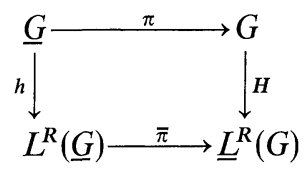

where $\bar{\pi}$ is the natural projection of $O\left(C^{p} \oplus R\right)$ on $O\left(M^{p}\right)$ and $h, H$ are the representation homomorphisms.

Proof. The proof follows the same steps of [2] for the quantum case. In each gauged ray $\underline{L}^{R}(A), A \in G$ we choose a canonical representative in $O\left(C^{p} \oplus R\right)$ in the form $\left(L^{R}(A), O\right)$. This is a well defined set of representatives since $\left[\left(L^{R}(A), O\right),\left(L^{R}(B), O\right)\right]=\left(L^{R}([A B]), O\right)$. Hence the Lie bracket of any representatives of the gauged rays in $O\left(C^{p} \oplus R\right)$ can be 
written in the form

$$
\begin{aligned}
& {\left[\left(L^{R}\left(A_{1}\right), d f_{1}\right),\left(L^{R}\left(A_{2}\right), d f_{2}\right)\right]=\left(L^{R}\left(\left[A_{1}, A_{2}\right]\right), d L^{R}\left(A_{1}\right) f_{2}\right.} \\
& \left.\quad-d L^{R}\left(A_{2}\right) f_{1}+d \xi\left(A_{1}, A_{2}, f_{1}, f_{2}\right)\right)
\end{aligned}
$$

where $\xi$ is a properly chosen factor i.e. the bracket operation is a Lie algebra bracket (we discuss the meaning of this condition in the next section).

We now define an extension $\underline{G}$ of $G$ as

$$
\underline{G}=\left\{(A, d f) ; A \in G, f \in C^{p-1}\right\}
$$

then $\underline{G}$ is a Lie algebra if we define the Lie bracket as follows

$$
\begin{aligned}
{\left[\left(A_{1}, d f_{1}\right),\left(A_{2}, d f_{2}\right)\right]=} & \left(\left[A_{1}, A_{2}\right], d L^{R}\left(A_{1}\right) f_{2}-d L^{R}\left(A_{2}\right) f_{1}\right. \\
& \left.+d \xi\left(A_{1}, A_{2}, f_{1}, f_{2}\right)\right)
\end{aligned}
$$

It is clear now that $\pi: \underline{G} \rightarrow G$ defined as $\pi(A, d f)=A$ is a projection of $\underline{G}$ onto $G$ and

$$
h:(A, d f) \rightarrow\left(L^{R}(A), d f\right)
$$

is a representation $L^{R}(\underline{G})$ of $\underline{G}$ in $O\left(C^{p} \oplus R\right)$ which coincide with $\underline{L}^{R}(G)$ on $M^{p}$. This proves our statement.

Remarks. 1. The theorem shows that any gauged representation of $G$ is "equivalent" to a representation of an infinite dimensional extension $\underline{G}$ of $G$. It follows therefore that the classification of the gauged representations of $G$ is equivalent to the classification of the possible $\xi$ 's and the representations of their associated $\underline{G}$.

2. Let $\left\{f_{a}\right\}$ be a basis of $C$ and denote the induced basis of $C^{p-1}$ by $F=\left\{f_{\lambda}\right\}$. Let $A_{i}$ be a basis of $G$ then it is obvious that $\left(A_{i}, d f\right)$ is a basis of $\underline{G}$.

If we now define

$$
\bar{A}_{i}=\left(A_{i}, 0\right), \quad \bar{f}_{\lambda}=\left(0, d f_{\lambda}\right)
$$

then $\underline{G}$ has the following commutation relations (assuming $\xi=\xi\left(A_{1}, A_{2}\right)$ )

$$
\begin{aligned}
& {\left[\bar{A}_{i}, \bar{A}_{j}\right]=\overline{\left[A_{i}, A_{j}\right]}+\bar{\xi}\left(A_{i}, A_{j}\right) .} \\
& {\left[\bar{f}_{\lambda}, \bar{f}_{\mu}\right]=0,} \\
& {\left[\bar{A}_{i}, \bar{f}_{\lambda}\right]=L^{R}\left(A_{i}\right) \bar{f}_{\lambda} .}
\end{aligned}
$$

To conclude this section we note that the analogy between the QuantumMechanical phase invariance and the gauge invariance of the Electromagnetic potentials is complete. In both cases this extra invariance leads to a natural generalization of the concept of Lie algebra representations and hence to extensions of the original Lie algebras. 


\section{The Generalized Bondi-Metzner Algebra}

In this section we specialize to an example which will illustrate the general theory related to the choice of the factors $\xi$ and have at the same time an independent physical interest. This example is the one gauged representations of the Lorentz algebra which are related to the Electromagnetic gauge invariance.

To accomplish this purpose we choose the differential manifold $X$ to be the two dimensional complex sphere [4] and a representation $R$ of the algebra on $C(X)$ induced by the action of the Lorentz group on $X$. The analysis of the preceeding section shows that in order to cope with the Electromagnetic gauge invariance we must consider one-gauged representations of the Lorentz algebra or stated differently we must consider representations on the one gauged rays $(L, d f), f \in C^{0}(X)$. The functions $f \in C^{0}(X)$ can be expanded into a series of spherical harmonics and therefore the one gauged rays in $M^{1}(X)$ can be written in the form

$$
\underline{L}=\left\{\left(L, \alpha_{l, m} d Y_{l, m}\right)\right\}
$$

where we used the convention that repeated indices are to be summed.

By Theorem 1 the classification of the one-gauged representations is equivalent to the classification of the factors $\xi\left(A_{i}, A_{j}\right)^{2}$ for which

$$
\begin{aligned}
& {\left[\left(L_{i}, \alpha_{l, m} d Y_{l, m}\right),\left(L_{j}, \beta_{l^{\prime}, m^{\prime}} d Y_{l^{\prime}, m^{\prime}}\right)\right]} \\
& \quad=\left(C_{i j}^{k} L_{k}, d\left(\beta_{l, m} L_{i} Y_{l, m}-\alpha_{l, m} L_{j} Y_{l, m}\right)+d \xi\left(Y_{l, m}, L_{i}, L_{j}\right)\right)
\end{aligned}
$$

is a Lie bracket (we dropped the superscript $R$ on the $L_{i}$ 's). This requirement imply immediately that $\xi$ is a bilinear antisymmetric form. However we must satisfy also the Jacobi identity which leads then to the equation

$$
\begin{aligned}
& d\left\{L_{k} \xi\left(Y_{l, m}, L_{i}, L_{j}\right)+L_{i}\left(Y_{l, m}, L_{j}, L_{k}\right)\right. \\
& \quad+L_{j} \xi\left(Y_{l, m}, L_{k}, L_{i}\right)+\xi\left(Y_{l, m}, L_{k},\left[L_{i}, L_{j}\right]\right) \\
& \left.\quad+\xi\left(Y_{l, m}, L_{i},\left[L_{j}, L_{k}\right]\right)+\xi\left(Y_{l, m}, L_{j},\left[L_{k}, L_{i}\right]\right)\right\}=0
\end{aligned}
$$

introducing the notation

$$
\begin{aligned}
\xi\left(Y_{l, m}, L_{i}, L_{j}\right) & =\beta_{i j}^{l, m} Y_{l, m} \\
L_{i} Y_{l, m} & =D_{l m, l^{\prime} m^{\prime}}^{i} Y_{l^{\prime} m^{\prime}} .
\end{aligned}
$$

Eq. (3.3) can be rewritten in the form

$$
\begin{aligned}
& {\left[\beta_{i j}^{l, m} D_{l, m, l^{\prime}, m^{\prime}}^{k}+\beta_{j, k}^{l, m} D_{l, m, l^{\prime}, m^{\prime}}^{i}+\beta_{k i}^{l, m} D_{l, m, l^{\prime}, m^{\prime}}^{j}\right.} \\
& \left.\quad+C_{i j}^{n} \beta_{k n}^{l^{\prime}, m^{\prime}}+C_{j k}^{n} \beta_{i n}^{l^{\prime}, m^{\prime}}+C_{k i}^{n} \beta_{j n}^{l^{\prime}, m^{\prime}}\right] d Y_{l^{\prime} m^{\prime}}=0 .
\end{aligned}
$$

\footnotetext{
${ }^{2}$ In principle $\xi=\xi\left(A_{1}, A_{2}, f_{1}, f_{2}\right)$ but we shall consider only the special case $\xi=\xi\left(A_{1}, A_{2}\right)$.
} 
Thus to classify the possible $\xi$ 's we must solve in general an infinite set of equations and we shall not attempt to tackle this problem here. A special solution of this system can be easily found however by setting $\beta_{i j}^{l, m}=0$.

For this choice of $\xi$ the structure of the one gauged algebra will be (in the notations of Eq. (2.20))

$$
\begin{aligned}
{\left[\bar{L}_{i}, \bar{L}_{j}\right] } & =C_{i j}^{k} \bar{L}_{k}, \\
{\left[\bar{Y}_{l, m}, \bar{Y}_{l^{\prime}, m^{\prime}}\right] } & =0, \\
{\left[\bar{L}_{i}, \bar{Y}_{l^{\prime} m^{\prime}}\right] } & =D_{l, m, l^{\prime}, m^{\prime}}^{i} \bar{Y}_{l^{\prime}, m^{\prime}}
\end{aligned}
$$

(when $\beta$ 三 0 (3.6a) will be replaced by

$$
\left[\bar{L}_{i}, \bar{L}_{j}\right]=C_{i j}^{k} \bar{L}_{k}+\beta_{i j}^{l, m} \bar{Y}_{l, m}
$$

which can be easily seen to be isomorphic to the generalized BondiMetzner algebra [4].

Thus we proved the following theorem:

Theorem 2. The Bondi-Metzner algebra is one of the possible algebras whose representations are equivalent to the one-gauged representations of Lorentz algebra. The consideration of these gauged representations is necessary in view of the gauge invariance of the Electromagnetic potentials.

Acknowledgements. It is a pleasure to thank Professor W. T. Sharp for several discussions. The hospitality extended to me at the University of Toronto is gratefully acknowledged.

\section{References}

1. Weyl, H.: The theory of groups and quantum mechanics. New York: Dover Publications 1950.

2. Bargmann, V.: Ann. Math. 59, 1 (1954).

3. Bondi, H., van der Burg, M.G. J., Metzner, A. W.K.: Proc. Roy. Soc. A 269, 21 (1962).

4. Sachs, R. K.: Phys. Rev. 128, 2851 (1962).

5. Newman,E.T., Penrose, R.: J. Math. Phys. 7, 863 (1966).

6. Flanders, H.: Differential forms. New York: Academic Press 1963.

Mayer Humi

Department of Mathematics

University of Toronto

Toronto 5, Canada 